\title{
Marker genes as predictors of shared genomic function
}

\author{
Joseph L. Sevigny ${ }^{1,3^{*}}$ iD, Derek Rothenheber ${ }^{1}$, Krystalle Sharlyn Diaz ${ }^{1,3}$, Ying Zhang ${ }^{2}$, Kristin Agustsson², \\ R. Daniel Bergeron ${ }^{2}$ and W. Kelley Thomas ${ }^{1,3}$
}

\begin{abstract}
Background: Although high-throughput marker gene studies provide valuable insight into the diversity and relative abundance of taxa in microbial communities, they do not provide direct measures of their functional capacity. Recently, scientists have shown a general desire to predict functional profiles of microbial communities based on phylogenetic identification inferred from marker genes, and recent tools have been developed to link the two. However, to date, no large-scale examination has quantified the correlation between the marker gene based taxonomic identity and protein coding gene conservation. Here we utilize 4872 representative prokaryotic genomes from NCBI to investigate the relationship between marker gene identity and shared protein coding gene content.

Results: Even at 99-100\% marker gene identity, genomes share on average less than 75\% of their protein coding gene content. This occurs regardless of the marker gene(s) used: V4 region of the 16S rRNA, complete 16S rRNA, or single copy orthologs through a multi-locus sequence analysis. An important aspect related to this observation is the intraorganism variation of $16 \mathrm{~S}$ copies from a single genome. Although the majority of $16 \mathrm{~S}$ copies were found to have high sequence similarity (>99\%), several genomes contained copies that were highly diverged ( $<97 \%$ identity).
\end{abstract}

Conclusions: This is the largest comparison between marker gene similarity and shared protein coding gene content to date. The study highlights the limitations of inferring a microbial community's functions based on marker gene phylogeny. The data presented expands upon the results of previous studies that examined one or few bacterial species and supports the hypothesis that $16 \mathrm{~S}$ rRNA and other marker genes cannot be directly used to fully predict the functional potential of a bacterial community.

Keywords: Metabarcoding, Metagenomics, 165 rRNA, Marker gene, Amplicon, Functional capacity, Comparative genomics

\section{Background}

Characterizing the diversity, abundance, and functional capacity of microbial communities has remained an important but difficult task for scientists. Current nextgeneration sequencing studies typically employ either full metagenome analysis, in which the entire genomic content of a community is sequenced, or marker gene analysis (also known as amplicon-based sequencing or metabarcoding) where individual genes, most often 16S rRNA, are targeted using amplification with conserved primers. While these

\footnotetext{
* Correspondence: jlsevigny1@wildcats.unh.edu

1 Molecular, Cellular, and Biomedical Sciences, University of New Hampshire,

46 College Rd, Rudman Hall, Durham, NH 03824, USA

${ }^{3}$ Hubbard Center for Genome Studies, University of New Hampshire, 35

Colovos Rd, Gregg Hall, Durham, NH 03824, USA

Full list of author information is available at the end of the article
}

amplicon-based studies provide valuable insight into the diversity and relative abundance of taxa within communities, they provide no direct insight into the function or genomic content of a community. Recently, there has been a surge in the desire to predict functional capacity based on taxonomic assignment from these amplicon studies. In fact, phylogeny has been used to infer the molecular functions of microbes in the past and in recent papers [1-3]. Tools such as PICRUSt [4] and Vikodak [5] have been created to link amplicon data to functional predictions. However, using phylogeny to predict functional content has two major limitations: it is largely dependent on database coverage [4], and it doesn't consider inputs from the local ecology (environmental conditions, taxa abundance, phage presence, etc.) in shaping community functions [6-8]. While authors are quick to acknowledge the limitations of

(c) The Author(s). 2019 Open Access This article is distributed under the terms of the Creative Commons Attribution 4.0 International License (http://creativecommons.org/licenses/by/4.0/), which permits unrestricted use, distribution, and reproduction in any medium, provided you give appropriate credit to the original author(s) and the source, provide a link to the Creative Commons license, and indicate if changes were made. The Creative Commons Public Domain Dedication waiver (http://creativecommons.org/publicdomain/zero/1.0/) applies to the data made available in this article, unless otherwise stated. 
inferred phylogeny to predict functional content, the limitations still exist.

There are multiple lines of evidence that $16 \mathrm{~S}$ rRNA is not an ideal marker for characterizing functional content [9-12]. One of the most well-known studies shows that three different strains of Escherichia coli (two pathogenic and one non-pathogenic) share less than $40 \%$ of their gene products, even though their $16 \mathrm{~S}$ sequences are identical [13]. Recent papers have also shown this heterogeneric relationship at the strain level with Roseobacter spp. and Microbacterium spp. [14, 15]. Thus, although at some level we already know the answer to this question, to date we have found no large-scale analysis to quantify the correlation between phylogenetic gene identity and functional capacity. An analysis of a broader spectrum of genomes has the potential to explore the more general limits of phylogenetic markers, such as $16 \mathrm{~S}$ rRNA, to predict community function.

This study aims to survey and quantify the variability of the 16S rRNA gene and select conserved single-copy ortholog genes (housekeeping genes) to examine its relationship with shared gene content. For this relationship, we hypothesized that the correlation would follow two general rates of change. (1) There is an unpredictable proportion of the genome typically acquired by horizontal gene transfer (conjunction, transformation, and transduction) [16], this proportion of the genome is independent of a phylogenic timescale, and results in an initial decrease of mean shared gene content between phylogenetically identical organisms. These events are likely 'random' or environmentally driven making accurate predictions impossible. (2) The remaining proportion of the genome consists of genes associated with 'core' function, and thus are conserved across phylogenetically related organisms. As phylogenetic distance increases there is a proportional decrease in shared gene content, likely due to slow gene loss, pseudogenes, and differences in genomic architecture $[17,18]$. Furthermore, we hypothesize that separate lineages/clades experience different rates of change, with respect to the adaptive and core genome. That is, certain lineages, such as E. coli and Vibrio spp., are more prone to and efficient in laterally transferring DNA, this results in a large pool of genes that are unshared between phylogenetically related organisms $[19,20]$. On the other hand, different lineages exhibit more of a genomic static state and result in a higher percentage of shared genes [21].

To test these hypotheses, we conducted a comparative genomic study using 4872 well-annotated prokaryotic reference genomes from the publicly available RefSeq database on NCBI (Fig. 1). Utilizing these genomes, we examined the relationship between 16S rRNA divergence and shared gene content on a large scale. We also examined the divergence of several concatenated single-copy orthologs to determine if they offer a means to combat

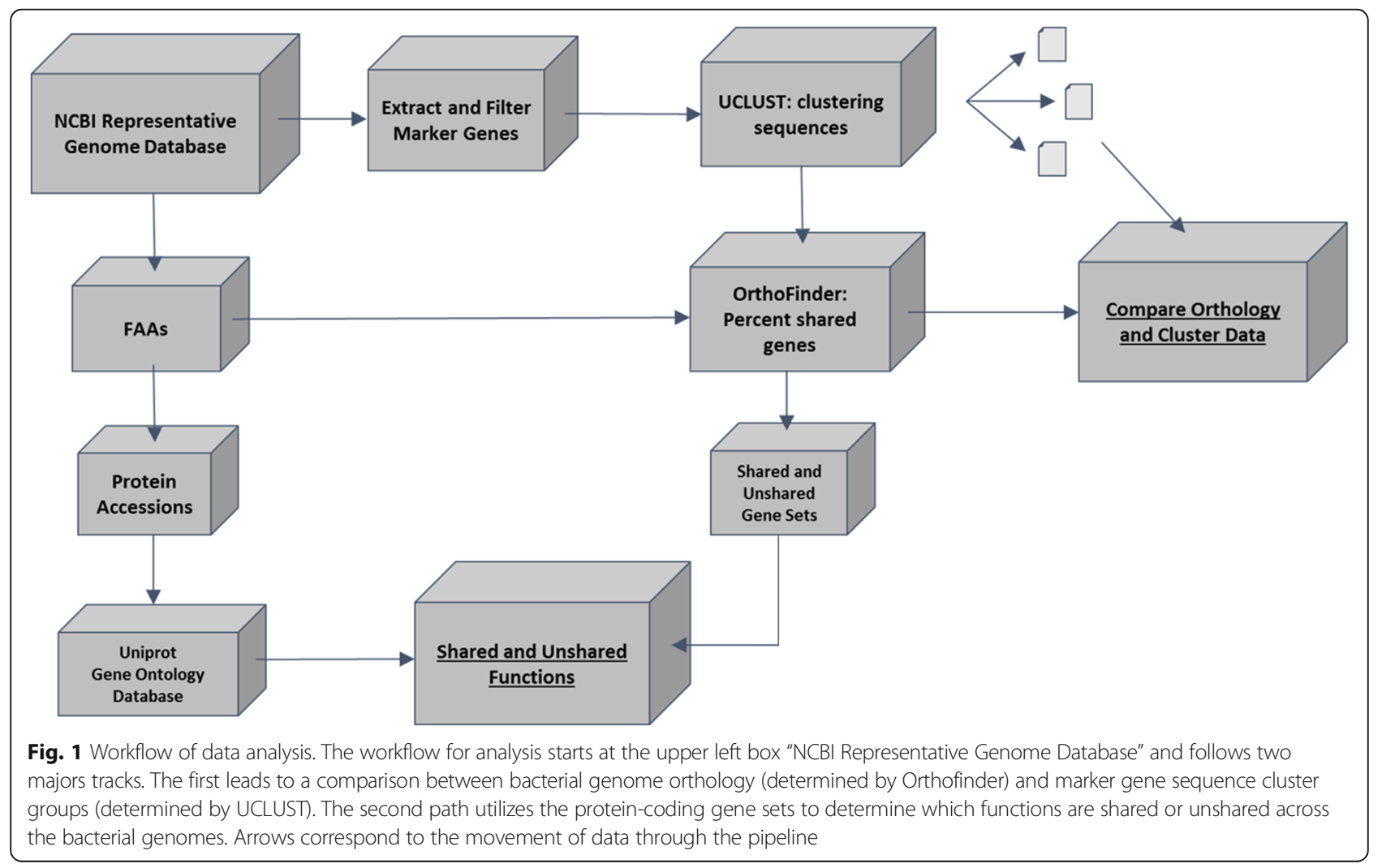


potential limitations in using the 16S rRNA. Lastly, we conducted a large-scale comparison between shared and novel gene sets to investigate the shared and novel functions of recently diverged prokaryotic organisms. Our goal was to determine aspects of the functional profile that may remain unknown when assuming a high predictable correlation between $16 \mathrm{~S}$ identity and organismal protein-coding gene content.

\section{Results}

\section{Prokaryote genome dataset and gene extraction}

A total of 4872 complete representative prokaryotic genomes are available through the NCBI ftp portal, spanning 28 of the 29 accepted bacterial phyla [22] and both classically accepted archaeal phyla, Crenarchaeota and Euryarcheaota [23]. Complete taxonomic distribution of the data, as constructed by Krona tools is shown in Fig. 2. See Additional file 1: Table S1 for a complete datasheet of all genome accessions and taxonomy used in this study. From this data, three amplicon datasets were generated: (1) Full-length $16 \mathrm{~S}$ rRNA, including 10,072 sequences from 4773 genomes, ranging in length between 1001 and $1856 \mathrm{bps}(\mu=1516.9, \sigma=86.5,2)$ The $\mathrm{V} 4$ region of the $16 \mathrm{~S}$ rRNA, including 9710 sequences from 4426 genomes, ranging in length between 334 and
509 bps ( $\mu=412.3, \sigma=4.6)$; and (3) Concatenated single-copy orthologs, including 3985 sequences (five genes), one for each genome, ranging in length between 6001 and $7434 \mathrm{bps}(\mu=7001.9, \sigma=376.5)$. In this multi-locus sequence analysis (MLSA) we chose five single-copy orthologs: $30 \mathrm{~S}$ ribosomal proteins S12 and S15, GTPase Der, ATP-synthase delta, and CTP synthase, because of their uniform presence and nomenclature across the dataset.

\section{Intra organism 16S rRNA variation}

For genomes within this dataset, 16S rRNA copy number ranged from one $(n=2485)$ to twenty $(n=1$, accession GCF_000686145), with an average of 2.3 copies per sample $(\sigma=2.1)$. The majority $(99.1 \%)$ of the $16 \mathrm{~S}$ rRNA copies with each genome examined have high sequence similarity ( $>97 \%$ ), however, a total of 38 genomes were found to have 16S rRNA copies that are less than 97\% identical (Fig. 3). See Additional file 2: Table S2 for the full datasheet of $16 \mathrm{~S}$ rRNA copy statistics. While no significant relationship between copy number and minimum gene identity was observed $\left(R^{2}=0.013\right)$, all genomes with less than $97 \%$ intra-genomic $16 \mathrm{~S}$ copy identity have less than nine copies of the gene.

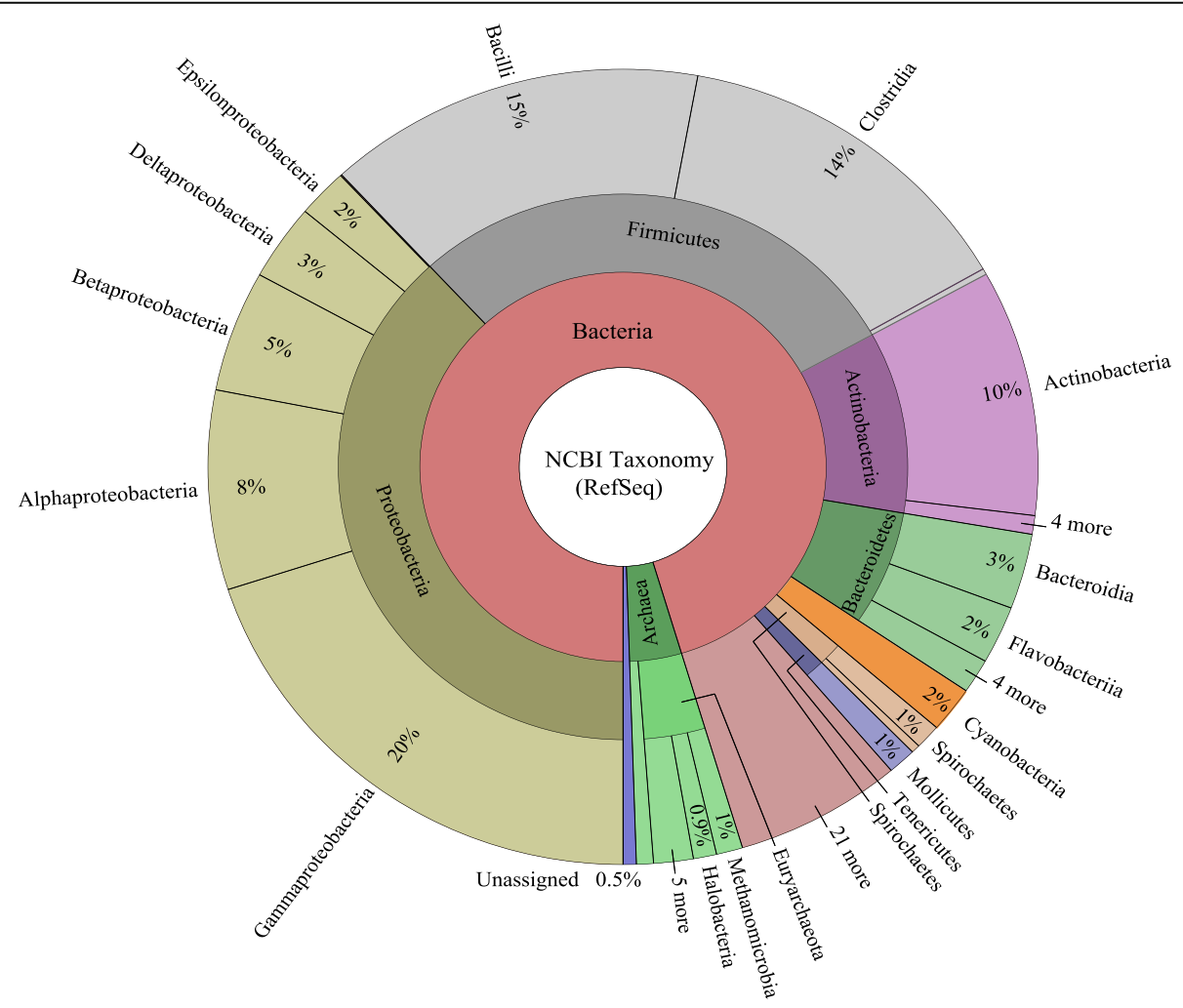

Fig. 2 Taxonomic classifications of NCBI's RefSeq representative prokaryotic genomes. A KronaTool map representing the relative taxonomic breakdown of the genomes used in this study. The inner circle represents genomes at the domain, the middle circle corresponds to phylum, and the outer circle represents data at the class level 


\section{Copy Number vs. Minimum Percent Identity}

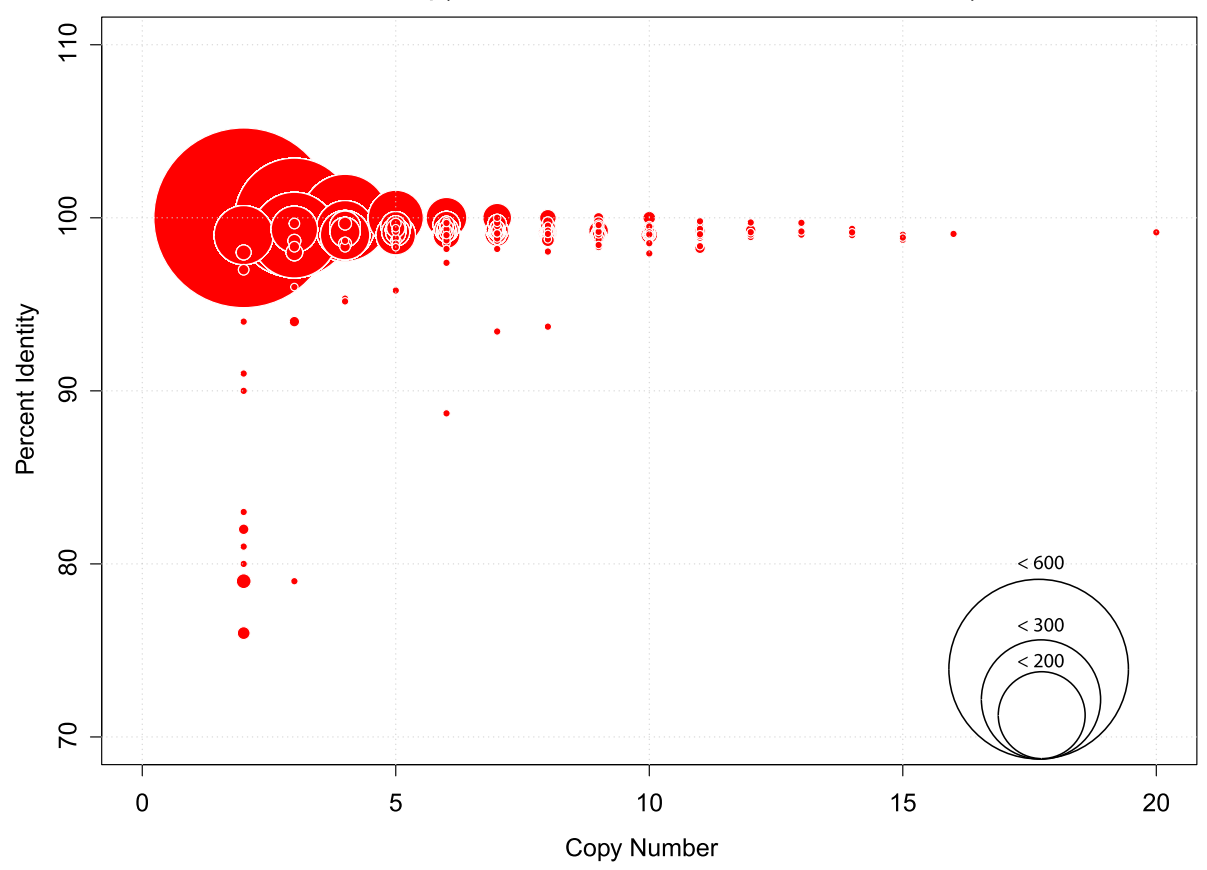

Fig. 3 Relationships between intra-organism 165 rRNA copy number and the percent identity across copies. A scatter bubble plot represents the relationship between 165 rRNA copy number and the percent identity between those copies. The circle size corresponds to the number of bacterial genomes with the same percent identity and copy number

Through sequence alignment validation of the low percent identity copies we found that 15 of the genomes contain one or more 16S rRNA copies with long stretches of unidentified nucleotides (represented by N's sequence) or had large gaps within the alignment. For example, the genome GCF_000332335 has five copies of the 16S rRNA gene, four of which were nearly identical while one sequence was highly diverged and contained several stretches of unidentified nucleotides. In such cases, these highly diverged copies were not included in the analyses comparing $16 \mathrm{~S}$ copy number and sequence variation.

\section{Sequence clustering}

To explore the relationship between gene identity and shared gene content, we first clustered all marker gene sequences at intervals between 95 and 100\%. This was completed separately for each of the three datasets (complete 16S, variable region of 16S, and MLSA). For the complete 16S rRNA and the V4 16S rRNA datasets, a large proportion of the $16 \mathrm{~S}$ rRNA copies from the genomes clustered with 16S rRNA copies from different genomes at or above $95 \%$ sequence similarity (71 and $80 \%$ respectively), thus retaining a large number of comparisons for this analysis. Because only $8 \%$ of the MLSA dataset concatenated sequences clustered with sequences from other genomes at $95 \%$ or greater, we included further comparisons at $93.0-93.9 \%$ and $94.0-94.9 \%$. At this range 520 marker gene sequences (13\%) clustered into groups with two or more unique genomes.

A representative graph depicting the sequence clustering of the complete 16S rRNA dataset for each percent identity group can be seen in Fig. 4 (a). The y-axis depicts the total number of $16 \mathrm{~S}$ rRNA clustering groups and the $\mathrm{x}$-axis depicts the total number of unique genomes (as represented by their 16S rRNA sequence) found within the respective clustering group. A similar trend was observed for each dataset. As the percent identity of the marker genes decreases there is an increase in marker gene clusters that include two or more genomes.

\section{Intra-organism 16S rRNA copies and sequence clustering}

As previously shown, many of the genomes in this dataset have low sequence similarity across their $16 \mathrm{~S}$ rRNA copies. To investigate how this affected sequence clustering we tracked the 16S rRNA copies for each genome across the clustering groups. Figure 4(c) and (d) depict the percentage of genomes whose 16S rRNA copies are found in one, two, or greater than two different $16 \mathrm{~S}$ rRNA clustering groups for the complete $16 \mathrm{~S}$ and V4 $16 \mathrm{~S}$ dataset respectively. As shown, the majority of the 16S rRNA copies from a single genome cluster into a single group, however, some are effectively divided and grouped with $16 \mathrm{~S}$ rRNA copies from a different genome. 


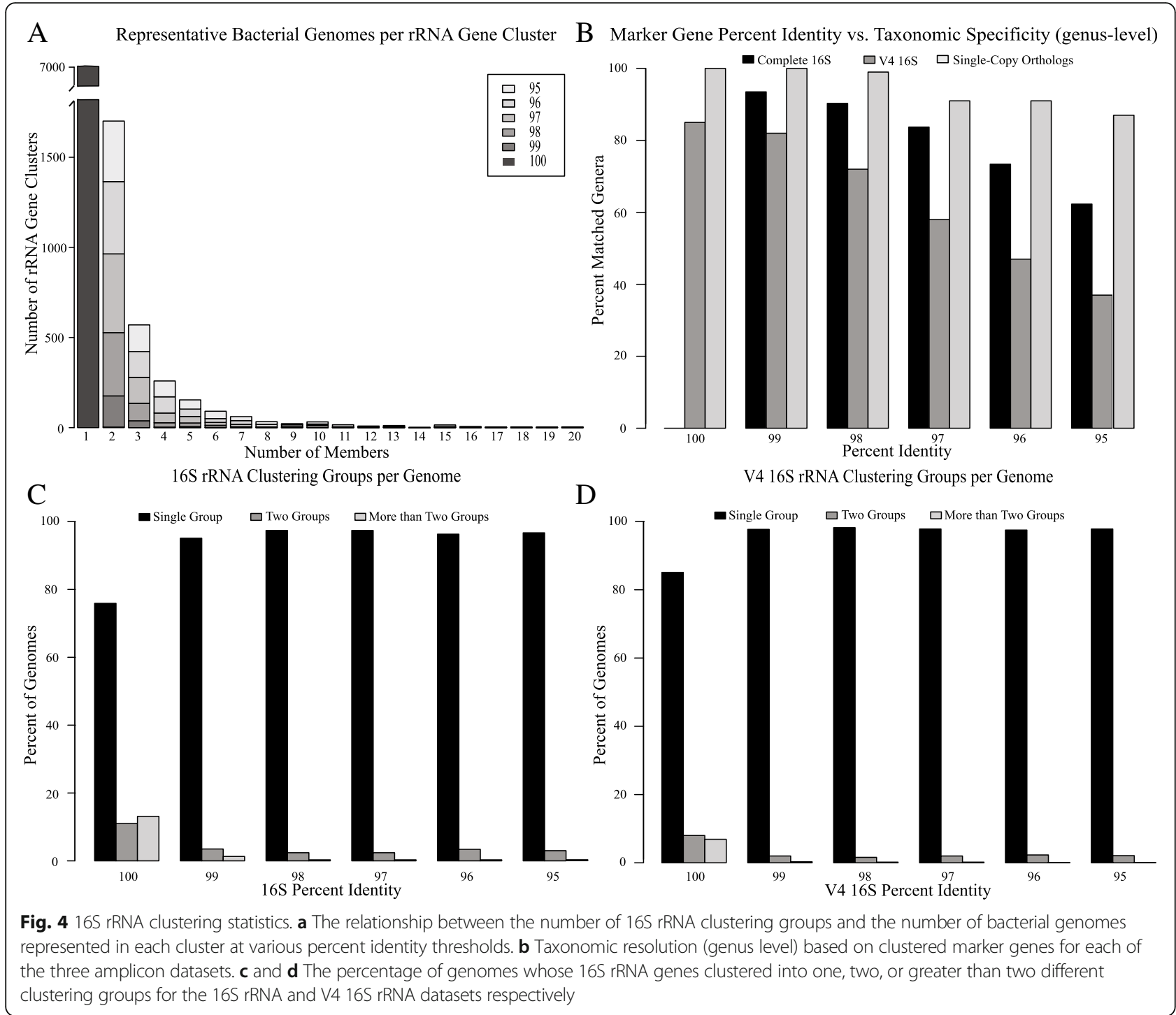

This is true regardless of the sequence identity threshold used.

\section{Marker gene sequence identity and taxonomic identification}

Determining proper phylogenetic identification is often an important step in metabarcoding analyses, we therefore examined the taxonomic relationships between genomes at various marker gene sequence identity thresholds. As shown in Fig. 4 (b), the MLSA-like approach shows the highest correlation between percent identity and taxonomic matches at the genus level. Even at $98 \%$ sequence similarity, $99 \%$ of the genomes cluster into groups with their respective genera. By contrast, genomes with 100\% V4 16S rRNA identity show only $85 \%$ taxonomic matches at the genus level and 58\% taxonomic matches at the genus level by $97 \%$ sequence identity.
Percent shared genes vs. marker gene similarity After clustering marker gene sequences into sequence similarity intervals, pairwise comparisons of protein coding gene content were completed for each genome using Orthofinder. Percent shared genes is defined here as the ratio between the number of genes matched among two genomes and the total number of genes present in both. Figure 5 depicts the relationship between similarity cutoff values and the percent shared gene content for the three different marker gene data sets. Among all comparisons, the percent shared genes range from 24.6 to 98.4\% and results show an initial decrease in shared genes between organisms whose marker genes cluster at $100 \%$ or $99 \%$. At the highest percent identity interval, the arithmetic means for each marker gene dataset are as follows; 78\% shared gene content at 99\% 16S rRNA identity, $72 \%$ shared gene content at $100 \%$ V4 16 S rRNA sequence identity, and $83 \%$ shared gene content at 100\% MLSA 

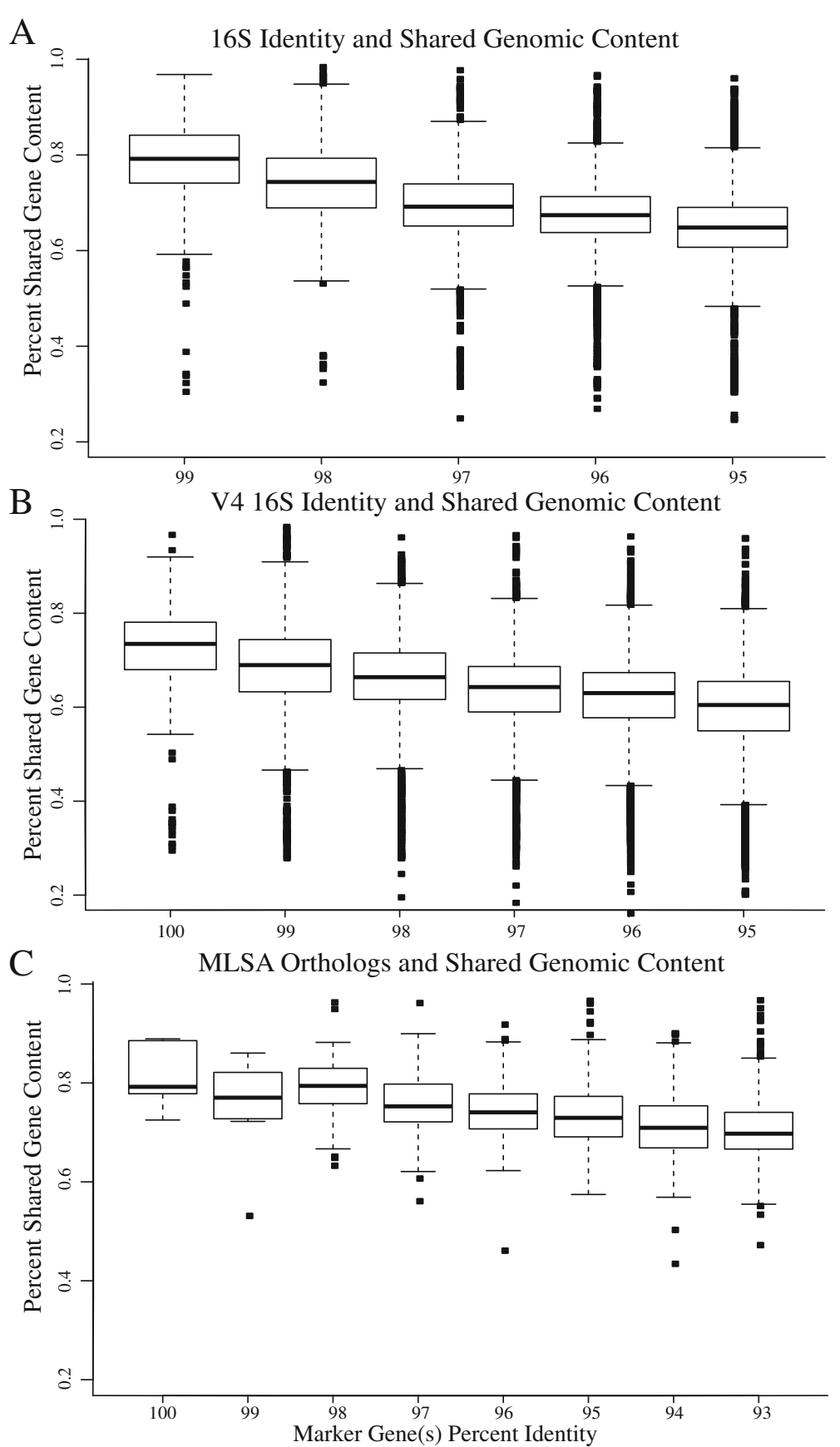

Fig. 5 Phylogenetic marker(s) and single-copy ortholog(s) relationship to shared gene content. Shown are box and whisker plots depicting the percentage of shared genes between genomes clustered at various percent identity intervals: (a) $16 \mathrm{~S}$ rRNA, (b) V4 16S rRNA, (c) Five-concatenated MLSA orthologs. Boxplots show the first and third quartile (bottom and top lines of the box), the median (middle line of the box), and the smallest and largest data-points excluding outliers (bottom and top whiskers). Data-points outside the whiskers correspond to outliers

sequence identity. While all datasets show a similar trend, the decrease in average shared gene content between cluster groups is highest in clustering done via the V4 $16 \mathrm{~S}$ rRNA (Fig. 5a) and lowest in the single-copy ortholog dataset (Fig. 5c). See Additional file 3: Table S3 for data used in construction of Fig. 5.
To expand upon these findings, we wanted to determine if there are certain groups of bacterial lineages where the relationship between marker gene identity and shared genome composition is higher or lower than the combined dataset (Fig. 6). We examined this in the V4 16S dataset at 99\% sequence similarity but expect 


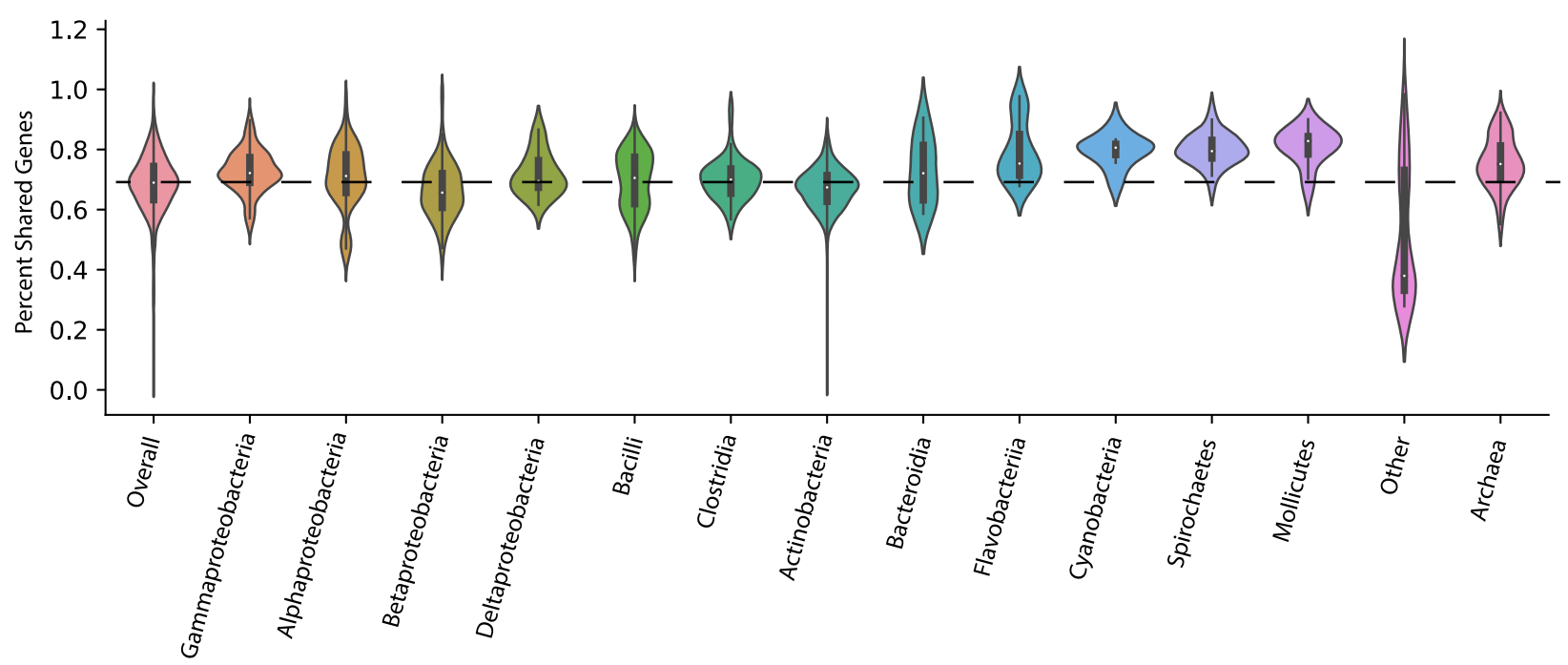

Fig. 6 Relationship between 99\% similar V4 16S rRNA and shared gene content across select microbial lineages. Violin plots representing the distribution of phylogenetically identical organisms (99\% V4 $16 \mathrm{~S}$ rRNA) across select microbial lineages and the percentage of shared gene content. The dotted black line corresponds to the mean shared gene content of the entire dataset and the width of the violin represents the relative concentration of data (i.e. wider regions contain more data points)

similar trends for other marker genes. Based on a Kruskal-Wallis test with a Dunn's multiple comparisons and Bonferroni correction the data shows that Spirochetes, Gammaproteobacteria, Cyanobacteria, Mollicutes, Archaea, and Flavobacteriia have a higher mean percent of shared genes $(p<0.05)$. The group termed "Other" is comprised of highly similar $16 \mathrm{~S}$ sequences that span different bacterial classes. As expected, these comparisons contain a significantly lower number of shared genes (Fig. 6; $p<0.05$ ). Other classes of bacteria, like Bacilli, Clostridia, and Alphaproteobacteria contain similar amounts of shared genes when compared to the overall dataset.

To further validate these findings and test if the unshared genes may have been misannotated or if their functionality was lost due to rapid evolution, we subjected unshared genes to a tBLASTn search against complete genomes in the clustering group rather than their associated protein FASTA file. We found that most of these genes $(\mu=87.4 \%, \sigma=0.3)$ are not found in the closely related genome at $>70 \%$ identity and only $1.8 \%$ on average are found at greater than 95\% identity (Additional file 4: Table S4).

\section{Shared and novel functions}

Data presented thus far shows genomes clustered via identical or near identical $16 \mathrm{~S}$ or single-copy orthologs share, on average, only $72-83 \%$ of protein-coding gene content. To determine if specific functions are more likely to appear in the shared or unshared across closely related bacterial genomes data sets, we analyzed the gene ontology (GO) of the matched and unmatched proteins identified from Orthofinder. We chose to focus on genomes whose V4 16S rRNA clustered at 99\% sequence similarity or greater. This dataset consists of $6,324,117$ protein accessions and 3515 total genome-genome comparisons. Of these accessions, 3,791,226 are found in the UniprotKB databases with a total of 2,803,829 containing gene ontology metadata. Results show 1794 GO terms significantly more likely to be shared, and 1119 GO terms more likely to be novel (unshared) ( $p$-value <0.01) (Additional file 5: Table S5). The top five significant shared and novel functions for each of the GO groups (biological process, molecular function, and cellular component) are shown in Table 1.

The top five GO terms (ordered by p-value) for each of the three broad categories of ontology: biological process, molecular function, and cellular component. 'Count-shared' and 'Count-unshared' refer to the number of times that particular GO term was found to be shared or unshared in the genome wide protein-coding content comparisons with Orthofinder. For an expanded summary of significant GO terms, see Table S5 in Additional file 5.

\section{Discussion}

\section{Dynamic genome evolution}

The current study quantifies the functional evolution of microbial genomes by describing the relationship between marker gene identity and shared protein-coding gene content. Results show that prokaryotic genomes exhibit a dynamic rate of evolutionary change. Although 
Table 1 Significant shared and unshared gene ontology terms between phylogenetically identical organisms (99\% V4 16S rRNA)

\begin{tabular}{|c|c|c|c|c|c|}
\hline Ontology & GO.ID & Term & Count-shared & Count-unshared & $P$-value \\
\hline \multicolumn{6}{|c|}{ Molecular Function } \\
\hline \multirow[t]{5}{*}{ unshared } & GO:0004803 & transposase activity & 4591 & 8641 & $<1 \mathrm{e}-30$ \\
\hline & GO:0003964 & RNA-directed DNA polymerase ... & 165 & 288 & $<1 \mathrm{e}-30$ \\
\hline & GO:0097351 & toxin-antitoxin pair type $\|$ bind ... & 72 & 274 & $<1 \mathrm{e}-30$ \\
\hline & GO:0090729 & toxin activity & 357 & 915 & $<1 \mathrm{e}-30$ \\
\hline & GO:0009036 & type II site-specific deoxyribon ... & 24 & 180 & $<1 \mathrm{e}-30$ \\
\hline \multirow[t]{5}{*}{ shared } & GO:0019843 & rRNA binding & 42,179 & 808 & $<1 \mathrm{e}-30$ \\
\hline & GO:0046872 & metal ion binding & 124,123 & 28,675 & $<1 \mathrm{e}-30$ \\
\hline & GO:0003735 & structural constituent of ribos ... & 63,194 & 2123 & $<1 \mathrm{e}-30$ \\
\hline & GO:0003723 & RNA binding & 32,770 & 4032 & $<1 \mathrm{e}-30$ \\
\hline & GO:0000287 & magnesium ion binding & 50,000 & 8454 & $<1 \mathrm{e}-30$ \\
\hline \multicolumn{6}{|c|}{ Biological Function } \\
\hline \multirow[t]{5}{*}{ unshared } & GO:0032196 & transposition & 1435 & 1887 & $<1 \mathrm{e}-30$ \\
\hline & GO:0045927 & positive regulation of growth & 69 & 327 & $<1 \mathrm{e}-30$ \\
\hline & GO:0045926 & negative regulation of growth & 86 & 338 & $<1 \mathrm{e}-30$ \\
\hline & GO:0051607 & defense response to virus & 235 & 756 & $<1 \mathrm{e}-30$ \\
\hline & GO:0043571 & maintenance of CRISPR repeat ... & 162 & 560 & $<1 \mathrm{e}-30$ \\
\hline \multirow[t]{5}{*}{ shared } & GO:0006412 & translation & 70,775 & 2978 & $<1 \mathrm{e}-30$ \\
\hline & GO:0071555 & cell wall organization & 18,821 & 1788 & $<1 \mathrm{e}-30$ \\
\hline & GO:0006457 & protein folding & 11,000 & 826 & $<1 \mathrm{e}-30$ \\
\hline & GO:0009252 & peptidoglycan biosynthetic proc. ... & 16,336 & 977 & $<1 \mathrm{e}-30$ \\
\hline & GO:0008360 & regulation of cell shape & 17,552 & 1049 & $<1 \mathrm{e}-30$ \\
\hline \multicolumn{6}{|c|}{ Cellular Component } \\
\hline \multirow[t]{5}{*}{ unshared } & GO:0012506 & vesicle membrane & 143 & 220 & $<1 \mathrm{e}-30$ \\
\hline & GO:0009341 & beta-galactosidase complex & 487 & 567 & $<1 \mathrm{e}-30$ \\
\hline & GO:0031469 & polyhedral organelle & 37 & 149 & $<1 \mathrm{e}-30$ \\
\hline & GO:0008305 & integrin complex & 38 & 83 & $<1 \mathrm{e}-30$ \\
\hline & GO:0030077 & plasma membrane light-harv ... & 68 & 147 & $<1 \mathrm{e}-30$ \\
\hline \multirow[t]{5}{*}{ shared } & GO:0015934 & large ribosomal subunit & 8067 & 252 & $<1 \mathrm{e}-30$ \\
\hline & GO:0005623 & cell & 15,927 & 4518 & $<1 \mathrm{e}-30$ \\
\hline & GO:0005886 & plasma membrane & 157,863 & 45,460 & $<1 \mathrm{e}-30$ \\
\hline & GO:0015935 & small ribosomal subunit & 7833 & 98 & $<1 \mathrm{e}-30$ \\
\hline & GO:0005737 & cytoplasm & 248,487 & 26,478 & $<1 \mathrm{e}-30$ \\
\hline
\end{tabular}

most of the genome mimics a rate of change following marker gene divergence, on average, $22-28 \%$ of the genome is independent of phylogenetic identity (Fig. 5). This dynamic nature can be explained by three general phenomena: 1) large introduction of non-native DNA from events like horizontal gene transfer; 2) gene deletion/loss of function; and 3) significant differences between genes in their evolutionary change. However, when we compared the unshared genes of genomes with high marker gene sequence similarity, we found that most of these genes have no matches even at low sequence identity thresholds, indicating that different rates of evolutionary change do not contribute significantly to genomic divergence in the tested genomes. We therefore propose that gene deletion, along with large introduction of non-native DNA, are more probable explanations for the results shown here. These phenomena are largely dependent on the organism's environment, resulting in a portion of the genome that is dependent on microbial niche, selective pressures, and environmental conditions [24-28]. 


\section{Choice of marker gene}

We analyzed the complete $16 \mathrm{~S}$ rRNA, the variable portion of the 16S rRNA, and various single-copy orthologs through an MLSA-like approach. We expected that the greater resolution by the complete $16 \mathrm{~S}$ dataset and MLSA approach would significantly dissect the observed rapid change in gene content, but it was only marginally improved. Although marginal, these results support the use of an MLSA approach to improve the resolving power between shared protein-coding gene content and percent identity. This style of analysis has been routinely used in genotyping pathogens, such as methicillin-resistant Staphylococcus aureus [29] or differentiating lineages or strains within a species $[30,31]$.

\section{Intra organism $16 \mathrm{~S}$ variation and genome clustering}

Evidence shown in both Fig. 3 and Fig. 4 (c and d) suggest that there is a subset of genomes with a few highly divergent copies of the 16S gene. While we observed that the majority of $16 \mathrm{~S}$ copies within a genome have high sequence similarity (>97\%), many contain 16 S copies with $>3 \%$ divergence, and similar findings have been reported in previous literature [32-34]. Furthermore, we observed that all genomes with greater than $3 \%$ divergence in $16 \mathrm{~S}$ copies are genomes with less than nine total copies (Fig. 3). Although untested here, this phenomenon may be an artifact of the assembly process, where sequences may become biased toward a consensus when deduced based on highly covered reads/kmers, such as those from genes with a high copy number. Conversely, this may reflect a mechanism of conserved evolution where genomes with greater copy numbers avoid unwarranted gene sequence changes via redundancy.

Figure 4 depicts how these divergent copies clustered within UCLUST. In cases where $16 \mathrm{~S}$ copies clustered into more than one group, some copies of the $16 \mathrm{~S}$ are more like copies in a different genome than $16 \mathrm{~S}$ copies within their own. In these cases, a single organism would be represented by several sequence variants and have a direct effect on functional prediction as well as affecting abundance estimations based on marker gene identity, even at the $97 \%$ species level criteria. Based on these observations a $97 \%$ criteria for clustering species is no more informative than $96 \%$ or $98 \%$. Even so clustering and predicting OTUs or assigning species level taxonomy based on $97 \%$ identity is practical and informative in most cases.

The field is moving away from using OTU sequence clustering for amplicon analyses and has begun to use exact sequence variants instead (i.e., 100\% sequence similarity clustering after error-correction) [35]. The results shown here mainly support this transition and expand upon the problems of using the typical 97\% OTU clustering for variant identification. For example, it is likely that OTUs are unnecessarily reducing our resolution by potentially grouping different genera into a single OTU (Fig. 5b).
However, using exact sequence variants (or 100\% OTU clustering) does not offer a means to combat the intra-organism 16S rRNA variation that often exists within an organism's genome (Figs. 4 and 5). This observation remains regardless of whether OTU clusters or exact sequence variants are used and remains a limitation with amplicon studies.

\section{Functional analysis}

The purpose of the GO enrichment analysis was to determine if the functions that change rapidly are unique and predictable. Additionally, we wanted to identify what functions are lost when a close correlation between marker gene identity and overall functional capacity is assumed. We found that although most gene ontology terms are shared across the genome dataset, many important and unique functions are significantly more prevalent in the novel/unshared gene sets (Table 1). Key functions such as 'transposase activity' (molecular function), 'transposition' (biological processes), and 'vesicle membrane' (cellular component) are the top hits across GO terms within this novel dataset. These processes may be related to horizontal gene transfer and represent key functions that could mediate microbial niche adaptation. Furthermore, many functions related to metabolic processes, such as 'glucosidase activity' or 'fucose metabolic processes', which may also be crucial to a specific environmental niche, are found in the unshared datasets. From the thousands of pairwise comparisons, we found that these functions are more likely to be found in unshared gene datasets. Within the shared datasets we observed GO terms such as 'DNA repair, 'DNA binding, and 'integral component of the plasma membrane'. These are essential components that are necessary for a microbe to function, regardless of environment.

These findings related to the novel/unshared PCG functions are expected and similar to the observation of a core and accessory genome within microbes and supports the pan-genome concept, which is the collection of shared genomic resources that varies across environments [36]. When scientists study the microbial community of a novel environment, they are often interested in how that community functions and differs from other known communities. By grouping species based on marker gene(s) sequence similarity and predicting functional content, we miss much of the novel functions or overestimate the functional capacity. This prevents thorough comparison of two communities and potentially hinders the discovery of novel functions, an aspect that may have motivated such a study in the first place.

\section{Dataset and potential bias}

The RefSeq representative prokaryotic genome database contains a large and diverse representation of major bacterial taxa for a comprehensive microbial dataset. All included genomes underwent a consistent annotation 
pipeline and nearly all protein-coding genes are linked to RefSeq GenBank files, so annotations and gene functions can be determined programmatically in an efficient manner. However, because many of the genomes available are biased towards biomedically and clinically relevant taxa, we anticipate some level of bias in the functional content of these organisms.

\section{Implications}

Authors of programs aimed at inferring functional content from amplicon data are quick to acknowledge the limitations that are expanded upon here. PICRUSt does provide a QC metric, Nearest Sequenced Taxon Index (NSTI), which can help elucidate the limitation of database coverage and aid in interpretation of data. However, databases such as GenBank are severely biased towards easily culturable bacteria, like Proteobacteria, which comprises of $46 \%$ of the genomes sequenced [37], leaving unculturable bacteria vastly uncharacterized. Depending on the environment sequenced, this could lead to a majority of bacterial functions being predicted from distantly related genomes [38]. Thus, programs such as PICRUSt and Vikodak promote a potentially misguided idea that the presence of certain organisms corresponds to what functions they should be carrying out. At best such programs can present hypotheses to be tested.

\section{Conclusions}

The central hypotheses in this study address the relationship between marker gene identity and protein coding gene content. We observed with overwhelming evidence that even phylogenetically identical organisms do not share substantial proportions of their gene products, highlighting the gap between marker gene identity and protein-coding gene content. Specifically, we found that $22-28 \%$ of an organism's functional capacity cannot be determined from marker gene(s) alone, even with MLSA. This is true even when analyzing $100 \%$ identical sequences, demonstrating the limitations of ampliconbased studies and their ability to characterize the functional capacity of microbial communities. Future studies using additional marker genes or other variable portions of the $16 \mathrm{~S}$ gene, along with environmental datasets, would build on the results presented here and further elucidate the dynamics of microbial evolution.

\section{Methods}

\section{Prokaryote genomes and $16 \mathrm{~S}$ extraction}

Prokaryotic genome and assembly accessions were identified from the NCBI representative genome report file. Corresponding genome/assembly FASTA, general feature format (GFF), amino acid FASTA (FAA), and GenBank feature format (GBFF) files were then downloaded via the NCBI ftp server ([39], release 75). Taxonomic information for each sample was determined from the README file within the ftp repository. Visualization of taxonomic information was completed with Krona tools v2.2 [40]. For each sample, a Python script was used to extract the 16S rRNA gene sequences from the genome assembly FASTA file. Gene identifications, direction, start, and stop locations were obtained directly from the corresponding GFF files. Sequences less than $1000 \mathrm{bps}$ in length were removed from the dataset and not included in subsequent steps. For each genome, $16 \mathrm{~S}$ copy number, sequence lengths, and intra-organism gene variation statistics were calculated. For genomes with two or more $16 \mathrm{~S}$ sequences, average and pairwise percent identity between 16S rRNA copies was determined using the T-Coffee v11.0 seq_reformat utility sim_idscore [41]. For genomes with two or more 16S rRNA copies that are less than $99.9 \%$ identical an alignment was constructed using Muscle v3.8.31 [42] and examined to validate the sequences and annotations.

\section{Extraction of the $16 \mathrm{~S}$ variable region}

An additional parallel dataset consisting of only the V4 variable region of each $16 \mathrm{~S}$ rRNA gene was also constructed. In this approach the $16 \mathrm{~S}$ variable region were extracted from each $16 \mathrm{~S}$ rRNA sequence bioinformatically using a pair of primers commonly used for amplicon studies, the 515f (GTGYCAGCMGCCGCGGTAA) forward primer and 926r (CCGYCAATTYMTTTRAGTTT) reverse primer.

\section{Single copy orthologs extraction and concatenation}

We constructed a third and final dataset consisting of concatenated single-copy orthologs to test a multi-locus sequence analysis (MLSA) like approach. Following the example of previous studies [43-45], single-copy orthologs present in at least $90 \%$ of bacterial species were identified using OrthoDB [29]. Out of the many potential genes identified, we chose five based on consistent annotation nomenclature and their presence as single copy genes across our dataset. For each organism, we extracted the five gene sequences from the genome assemblies and then concatenated them into a single sequence.

\section{Clustering gene sequences}

For each of the three datasets (full-length 16S, V4 16S, and MLSA), we used UCLUST software v1.2.22q [46] to cluster the prokaryotic sequences into a set of clusters based upon sequence similarity. We clustered the sequences using identity thresholds of 95, 96, 97, 98, 99, and $100 \%$ to discern meaningful trends.

To investigate how the 16S rRNA gene copies from a single genome fell out into clustering groups, we examined the number of unique clustering groups per genome and identified any genomes whose rRNA copies were 
found in different clustering groups. In addition, we identified the number of unique genomes represented in each clustering group and their taxonomic assignments.

\section{Calculating percent shared genes}

Next, we wanted to calculate the shared gene content between all genomes represented within the marker gene clustering groups using the program OrthoFinder v0.4, with default settings [47]. For validation of this method we subjected unmatched genes identified in Orthofinder to a tBLASTn search against the complete genomes of other members in the respective cluster. We recorded significant matches (e-value $<1 \mathrm{e}-10)$ with a query coverage and percent identity greater than $70 \%$.

The shared gene content comparisons were then linked back to the marker gene clustering groups obtained from UCLUST. Each comparison is only included in the highest percent identity group, effectively dividing the data into comparisons from 95.0-95.99, 96.0-96.99, 97.0-97.99, 98.0-98.99, 99.0-99.99, and 100\% marker gene identity. Using $\mathrm{R}$ v2,14.2, we created box plots depicting shared content in relation to percent marker gene identity for each of the three datasets.

To determine if different lineages exhibit a higher or lower relationship between shared gene content and marker gene percent identity compared to the complete dataset, we split the V4 16S dataset into each of the major bacterial classes and completed the marker gene clustering and percent shared gene calculations outlined above. A Kruskal-Wallis test followed by a Dunn test for multiple comparisons with a Bonferroni correction was then completed to determine if the mean percent shared genes for each lineage was significantly different than the complete dataset.

\section{Determining shared and novel functions}

To better understand the differences in shared and novel functions of closely related genomes, we examined all protein-coding genes from genomes whose V4 $16 \mathrm{~S}$ clustered together at $99 \%$ identity. First, the count of each protein accession within a matched or unmatched Orthofinder output file was determined across all comparisons. We linked accessions to gene ontology (GO) using the UniprotKB Swiss-Prot and TrEMBL databases (download date: May 01, 2016). Protein accessions and their respectively mapped GO terms were imported into the topGO software v3.8 [48]. For each gene ontology environment (molecular function, biological process, cellular component) enrichment of matched and unmatched GO's were tested using Fisher's exact test with the 'weight01' algorithm.

\section{Statistical analyses}

All routine statistical analyses were performed in either Python v3.4 or R v2.14.2 with plottrix package [49].

\section{Additional files}

Additional file 1: Table S1. The table provides the genome accessions and taxonomy for all reference sequences used in this study. (XLSX $169 \mathrm{~kb}$ )

Additional file 2: Table S2. Summary of $16 S$ rRNA copy statistics for each bacterial genome. Included is the number of $16 \mathrm{~S}$ rRNA copies, the minimum and maximum length among copies, the average length of copies, and the minimum and maximum percent identity among copies. (XLSX $93 \mathrm{~kb}$ )

Additional file 3: Table S3. A list showing the ratio of shared genes for each bacterial genome comparison. Data is included for each of the three datasets; Full-length 165 rRNA, V4 165 rRNA, and MLSA. (XLSX 1048 kb)

Additional file 4: Table S4. Summary of BLAST results comparing the unshared gene content of bacterial genomes whose V4 16s rRNA clustered at 99\% identity. (XLSX $23 \mathrm{~kb}$ )

Additional file 5: Table S5. The table provides details of all significantly shared and unshared GO terms for bacterial genomes whose V4 $16 \mathrm{~S}$ rRNA sequences clustered at greater than 99\% identity. (XLSX 158 kb)

\section{Abbreviations}

16S rRNA: $16 S$ Ribosomal RNA; BLAST: Basic Local Alignment Search Tool; E. coli: Escherichia coli; GO: Gene Ontology; MLSA: Multilocus Sequence Analysis; NCBI: National Center for Biotechnology Information; NSTI: Nearest Sequenced Taxon Index; OTU: Operational Taxonomic Unit; RefSeq: Reference Sequence Database; tBLASTn: Protein-Nucleotide 6-frame translation (BLAST)

\section{Acknowledgements}

Not applicable.

\section{Funding}

We would like to thank the Ocean Leadership/Gulf of Mexico Research Initiative (GOMRI) Grant 16-052 (to W.K.T) (SA 16-18) and the National Institutes of Health New Hampshire Idea Network of Biological Research Excellence (5 P20 GM 10350605) for funding and support. In consideration of partial funding by GOMRI, data are publicly available through the Gulf of Mexico Research Initiative Information \& Data Cooperative (GRIIDC) at https://data.gulfresearchinitiative.org (https://doi.org/10.7266/n7-tc8j-1562).

\section{Availability of data and materials}

The scripts and datasets generated and/or analyzed during the current study are archived with Zenedo at https://doi.org/10.5281/zenodo.1309979, available at https://github.com/Joseph7e/Marker-genes-as-predictors-of-shared-genomicfunction., and the Gulf of Mexico Research Initiative Information \& Data Cooperative (GRIIDC) at https://data.gulfresearchinitiative.org (doi: doi:https://doi. org/10.7266/n7-tc8j-1562). All starting data is publicly available from the NCBI representative microbial genome ftp site at ftp:/ftp.ncbi.n/m.nih.gov/genomes/.

\section{Authors' contributions}

JS, DR, RDB, WKT, designed research. WKT, contributed with funding. JS, DR, KSD, YZ, KA, contributed to programming and data analysis. JS, DR final data analyses and wrote the paper with the help of all the other authors. All authors read and approved the final manuscript.

\section{Ethics approval and consent to participate}

Not applicable.

\section{Consent for publication}

Not applicable.

\section{Competing interests}

The authors declare that they have no competing interests.

\section{Publisher's Note}

Springer Nature remains neutral with regard to jurisdictional claims in published maps and institutional affiliations. 


\section{Author details}

${ }^{1}$ Molecular, Cellular, and Biomedical Sciences, University of New Hampshire, 46 College Rd, Rudman Hall, Durham, NH 03824, USA. ²Department of Computer Science, University of New Hampshire, 33 Academic Way, Kingsbury Hall, Durham, NH 0324, USA. ${ }^{3}$ Hubbard Center for Genome Studies, University of New Hampshire, 35 Colovos Rd, Gregg Hall, Durham, $\mathrm{NH}$ 03824, USA.

Received: 12 July 2018 Accepted: 24 March 2019

Published online: 04 April 2019

\section{References}

1. Lesser MP, Fiore C, Slattery M, Zaneveld J. Climate change stressors destabilize the microbiome of the Caribbean barrel sponge, Xestospongia muta. J Exp Mar Biol Ecol. 2016:475:11-8.

2. Peter H, Sommaruga R. 2016. Shifts in diversity and function of lake bacterial communities upon glacier retreat. ISME J. 2016;10:1545-54.

3. Salles JF, Le Roux X, Poly F. (2015). Relating phylogenetic and functional diversity among denitrifiers and quantifying their capacity to predict community functioning. Front Microbiol. 2015;3. https://doi.org/10.3389/ fmicb.2012.00209.

4. Langille MG, Zaneveld J, Caporaso JG, McDonald D, Knights D, Reyes JA, et al. Predictive functional profiling of microbial communities using $16 \mathrm{~S}$ rRNA marker gene sequences. Nature Biotechnol. 2013;31:814-21.

5. Nagpal S, Haque MM, Mande SS. Vikodak - a modular framework for inferring functional potential of microbial communities from $16 \mathrm{~S}$ metagenomic datasets. PLoS One. 2016;11(2):e0148347. https://doi.org/10. 1371/journal.pone.0148347.

6. Berg G, Smalla K. Plant species and soil type cooperatively share the structure and function of microbial communities in the rhizosphere. FEMS Microbiol Ecol. 2009;68:1-13 https://doi.org/10.1111/j.1574-6941.2009.00654.x.

7. Koskella B, Brockhurst MA. Bacteria-phage coevolution as a driver of ecological and evolutionary processes in microbial communities. FEMS Microbiol Rev. 2014;38:916-31. https://doi.org/10.1111/1574-6976.12072.

8. Smillie CS, Smith MB, Friedman J, Cordero OX, David LA, Alm EJ. Ecology drives a global network of gene exchange connecting the human microbiome. Nature. 2011;280. https://doi.org/10.1038/nature10571.

9. Coleman ML, Sullivan MB, Martiny AC, Steglich C, Barry K, DeLong EF, Chisholm SW. Genomic islands and the ecology and evolution of Prochlorococcus. Science. 2011;311:1768-70.

10. Brochet M, Rusniok C, Couvé E, Dramsi S, Poyart C, Trieu-Cuot P, Kunst F, Glaser $P$. Shaping a bacterial genome by large chromosomal replacements, the evolutionary history of Streptococcus agalactiae. Proc Natl Acad Sci U S A. 2008:105:15961-6.

11. Earl AM, Losick R, Kolter R. Ecology and genomics of Bacillus subtilis. Trends Microbiol. 2008;16:269-75.

12. Deurenberg RH, Stobberingh EE. The evolution of Staphylococcus aureus. Infect Genet Evol. 2008:8:747-63.

13. Welch RA, Burland V, Plunkett GIII, Redford P, Roesch P, Rasko D, Buckles EL, Liou SR, Boutin A, Hackett K, Stroud D, Mayhew GF, Rose DJ, Zhou S, Schwartz DC, Perna NT, Mobley HL, Donnerberg MS, Blattner FR. Extensive mosaic structure revealed by the complete genome sequence of uropathogenic Escherichia coli. Proc Natl Acad Sci U S A. 2002;99:17020-4

14. Zhang $Y$, Sun $Y$, Jiao N, Stepanauskas R, Luo H. Ecological genomics of the uncultivated marine Roseobacter lingage CHAB-I-5. Appl Envir Microbiol. 2016;82. https://doi.org/10.1128/AEM.03678-15.

15. Henson MW, Santo Domingo JW, Kourtev PE, Jensen RV, Dunn JA, Learman DR. Metabolic and genomic analysis elucidates strains-level variation in Microbacterium spp. isolated from chromate contaminated sediment. PeerJ. 2015;10. https://doi.org/10.7717/peerj.1395.

16. Pál C, Papp B, Lercher MJ. Adaptive evolution of bacterial metabolic networks by horizontal gene transfer. Nat Genet. 2005;31:1372-5.

17. Kuo $\mathrm{CH}$, Ochman $\mathrm{H}$. The extinction dynamics of bacterial pseudogenes. PLoS Genet. 2010;6. https://doi.org/10.1371/journal.pgen.1001050.

18. Lawrence JG, Hendrickson H. Genome evolution in bacteria: order beneath chaos. Curr Opin Microbiol. 2005;8:572-8.

19. Dutilh BE, Cassman N, McNair K, Sanchez SE, Silva GZ, Boling L, Barr JJ, Speth DR, Seguritan V, Aziz RK, Felts B, Dinsdale EA, Mokili JL, Edwards RA. A highly abundant bacteriophage discovered in the unknown sequences of human faecal metagenomes. Nat Commun. 2014;5. https://doi.org/10.1038/ ncomms5498.
20. Lukjancenko O, Wassenaar TM, Ussery DW. Comparison of 61 sequenced Escherichia coli genomes. Microb Ecol. 2010;60. https://doi.org/10.1007/ s00248-010-9717-3.

21. Van Ham RC, Kamerbeek J, Palacios C, Rausell C, Abascal F, Bastolla U, Fernández JM, Jiménez L, Postigo M, Silva FJ, Tamames J, Viguera E, Latorre A, Valencia A, Morán F, Moya A. Reductive genome evolution in Buchnera aphidicola. Proc Natl Acad Sci U S A. 2003;100:581-6.

22. Brown CT, Hug LA, Thomas BC, Sharon I, Castelle CJ, Singh A, et al. (2015). Unusual biology across a group comprising more than $15 \%$ of domain Bacteria. Nature. 523: 208-211.

23. Yarza P, Yilmaz P, Pruesse E, Glöckner FO, Ludwig W, Schleifer KH, Whitman WB, Euzéby J, Amann R, Rosselló-Móra R. Uniting the classification of cultured and uncultured bacteria and archaea using 165 rRNA gene sequences. Narure Rev Microbiol. 2014;12:635-45. https://doi.org/10.1038/ nrmicro3330.

24. Cooper VS, Vohr SH, Wrocklage SC, Hatcher PJ. Why genes evolve faster on secondary chromosomes in bacteria. PLoS Comput Biol. 2010;6 https://doi. org/10.1371/journal.pcbi.1000732.

25. Doolittle WF, Zhaxybayeva O. On the origin of prokaryotic species. Genome Res. 2009;19:744-56. https://doi.org/10.1101/gr.086645.108.

26. Lawrence JG. Gene transfer in bacteria: speciation without species? Theor Popul Biol. 2002;61:449-60.

27. Polz MF, Alm EJ, Hanage WP. Horizontal gene transfer and the evolution of bacterial and archaeal population structure. Trends Genet. 2013;29:170-5.

28. Retchless AC, Lawrence JG. Temporal fragmentation of speciation in bacteria. Science. 2007;317:1093-6.

29. Enright MC, Robinson DA, Randle G, Feil EJ, Grundmann H, Spratt BG. The evolutionary history of methicillin-resistant Staphylococcus aureus (MRSA). Proc Natl Acad Sci U S A. 2002;99:7687-92.

30. Maiden MC, van Rensburg MJJ, Bray JE, Earle SG, Ford SA, Jolley KA, et al. MLST revisited: the gene-by-gene approach to bacterial genomics. Nat Rev Microbiol. 2013;11:728-36. https://doi.org/10.1038/nrmicro3093.

31. Xu F, Ilyas S, Hall JA, Jones SH, Cooper VS, Whistler CA. Genetic characterization of clinical and environmental Vibrio parahaemolyticus from the Northeast USA reveals emerging resident and non-indigenous pathogen lineages. Front Microbiol. 2015;6 https://doi.org/10.3389/fmicb.2015.00272

32. Pei AY, Oberdorf WE, Nossa CW, Agarwal A, Chokshi P, Gerz EA, Jin Z, Lee P, Yang L, Poles M, Brown SM, Sotero S, DeSantis T, Brodie E, Nelson K, Pei Z. Diversity of $16 \mathrm{~S}$ rRNA genes within individual prokaryotic genomes. Appl Environ Microbiol. 2010;76:3886-97. https://doi.org/10.1128/AEM.02953-09.

33. Stackebrandt E, Goebel BM. Taxonomic note: a place for DNA-DNA reassociation and 165 rRNA sequence analysis in the present species definition in bacteriology. Int J Syst Evol Microbiol. 1994;44:846-9.

34. Vètrovský $T$, Baldrian P. The variability of the 165 rRNA gene in bacterial genomes and its consequences for bacterial community analyses. PLoS One. 2013;8(2):e57923. https://doi.org/10.1371/journal.pone.0057923.

35. Callahan BJ, McMurdie PJ, Rosen MJ, Han AW, Johnson AJ, Holmes SP. DADA2: high-resolution sample inference from Illumina amplicon data. Nat Methods. 2016:15:581-3.

36. Fullmer MS, Soucy SM, Gogarten JP. The pan-genome as a shared genomic resource: mutual cheating, cooperation and the black queen hypothesis. Front Microbiology. 2015;6. https://doi.org/10.3389/fmicb.2015.00728.

37. Land M, Hauser L, Jun SR, Nowkaew I, Leuze MR, Ahn TH, Lund O, Kora G, Wassenaar T, Poudel S, Ussery DW. Insights from 20 years of bacterial genome sequencing. Funct Integr Genomics. 2015;2:141-61. https://doi.org/ 10.1007/s10142-015-0433-4.

38. Aßhauer KP, Wemheuer $B$, Daniel $R$, Meinicke P. Tax4Fun: predicting functional profiles from metagenomic 165 rRNA data. Bioinformatics. 2015;31:2882-4

39. The NCBI handbook [Internet]. Bethesda: National Library of Medicine (US), National Center for Biotechnology Information; 2002 Oct. Chapter 18, The Reference Sequence (RefSeq) Project. Available from http://www.ncbi.nlm nih.gov/books/NBK21091. Accessed 7 May 2016.

40. Ondov BD, Bergman NH, Phillippy AM. Interactive metagenomic visualization in a web browser. BMC Bioinformatics. 2011;12:1-9.

41. Notredame C, Higgins DG, Heringa J. T-coffee: a novel method for fast and accurate multiple sequence alignment. J Mol Biol. 2000;302:205-17.

42. Atkinson GC. The evolutionary and functional diversity of classical and lesser-known cytoplasmic and organellar translational GTPases across the tree of life. BMC Genomics. 2015;16:1-15. https://doi.org/10.1186/s12864015-1289-7. 
43. Gao B, Gupta RS. Phylogenetic framework and molecular signatures for the main clades of the phylum Actinobacteria. Microbiol Mol Biol Rev. 2012;76:66-112.

44. Lang JM, Darling AE, Eisen JA. Phylogeny of bacterial and archaeal genomes using conserved genes: supertrees and supermatrices. PLoS One. 2013;8(4): e62510. https://doi.org/10.1371/journal.pone.0062510.

45. Kriventseva EV, Tegenfeldt F, Petty TJ, Waterhouse RM, Simão FA, Pozdnyakov IA, loannidis P, Zbobnov EM. OrthoDB v8: update of the hierarchical catalog of orthologs and the underlying free software. Nucleic Acids Res. 2015;43:250-6. https://doi.org/10.1093/nar/gku1220.

46. Edgar RC. Search and clustering orders of magnitude faster than BLAST. Bioinformatics. 2010;26:2460-1.

47. Emms DM, Kelly S. OrthoFinder: solving fundamental biases in whole genome comparisons dramatically improves orthogroup inference accuracy. Genome Biol. 2015;16:1-14.

48. Alexa A, Rahnenfuhrer J. topGO: Enrichment analysis for gene ontology. R package version 2.34.0. 2018.

49. Lemon J. Plotrix: A package in the red light district of R. R-news. 2006;6:8-12.

Ready to submit your research? Choose BMC and benefit from:

- fast, convenient online submission

- thorough peer review by experienced researchers in your field

- rapid publication on acceptance

- support for research data, including large and complex data types

- gold Open Access which fosters wider collaboration and increased citations

- maximum visibility for your research: over $100 \mathrm{M}$ website views per year

At $\mathrm{BMC}$, research is always in progress.

Learn more biomedcentral.com/submissions 